\title{
Il Cinema VR come esperienza immersiva archi-schermica
}

\author{
Francesco Melchiorri \\ Ricercatore indipendente
}

\begin{abstract}
Il saggio intende proporre una riflessione teorica nei confronti del Cinema VR, pratica artistica recente che ha visto la luce applicando l'apparato tecnico ed estetico della Virtual Reality alla narratività e agli stilemi cinematografici. Dopo una breve premessa etimologica attorno all'idea di immersività, utile a circoscrivere il campo d'indagine, viene introdotto l'oggetto di studio suddetto, cercando di analizzarne la natura mediale e descrivendo perciò quest'ultima attraverso alcune riflessioni di Citton e di Auslander. Ci si sofferma in seguito sul pensiero di Carbone e sulla sua "filosofia-schermi", tentando di associare al Cinema VR I'“idea sensibile" di "archi-schermo", attraverso un discorso sulla percezione tra corpo e schermo. Infine, dopo alcune precisazioni di carattere tecnico, si cerca di rilanciare il discorso teorico appoggiandosi all'analisi di D'Aloia, cercando di esplicitare quanto sviluppato lungo la trattazione applicandolo, evidenziandone così da ultimo anche possibili ampliamenti futuri.
\end{abstract}

The essay aims to propose a theoretical reflection about VR Cinema, a recent artistic practice born by applying the technical and aesthetic apparatus of Virtual Reality to narrative and stylistic features of Cinema. After a brief etymological premise around the idea of immersivity, useful for circumscribing the field of investigation, we will introduce the Cinema VR, trying to analyse its media nature describing it through some reflections by Citton and by Auslander. We then will focus on Carbone's "philosophy-screens", trying to associate the "sensitive idea" of "arche-screen" with the Cinema VR, through a discussion on the perception between body and screen. In the end, after some clarifications of a technical nature, we will return to the theoretical exploration using the analysis of D'Aloia. In this way we will try to explain what we developed throughout the discussion, highlighting also possible future extensions.

\section{Parole chiave/Key Words}

Immersività; Cinema VR; CARNE y ARENA; Mauro Carbone; archi-schermo.

Immersivity; VR Cinema; CARNE y ARENA; Mauro Carbone; arche-screen. 


\section{Immersività e medialità}

\section{Cosa si intende per immersività?}

Secondo il vocabolario online Treccani con "immersivo", "[d]erivato dal p. pass. e agg. immerso con l'aggiunta del suffisso -ivo, per influsso dell'agg. ingl. immersive ${ }^{1}{ }^{1}$, si indica un aggettivo riferibile a un ambiente, un luogo reale o virtuale «[n]el quale si entra completamente, rimanendone avvolti e catturati $»^{2}$. Per quanto coerente con il verbo italiano da cui deriva, è proprio nel suo originale inglese però che il termine acquista un significato più sfaccettato e al contempo preciso. Nel Cambridge Dictionary alla voce "immersive" troviamo l'afferenza, in qualità di aggettivo, al registro proprio dei «media, theatre \& film», seguito immediatamente dalla definizione: «[che] sembra circondare il pubblico, l'agente, ecc. in modo che si sentano completamente coinvolti in qualcosa» ${ }^{3}$. Siamo quindi passati da una situazione in cui si è «avvolti e catturati» a un'altra dove ci si sente «completamente coinvolti in qualcosa». Se il primo caso denota una passività del soggetto nel subire l'azione di cui si parla, diversamente essere "coinvolti" in qualcosa sembrerebbe sottintendere un coinvolgimento attivo (sia esso fisico, emotivo, mentale) nella situ-azione, vale a dire nell'azione specificatamente ubicata. Nella lingua inglese, inoltre, l'aggettivo "immersive" descrive all'interno della terminologia computazionale ciò che fornisce informazioni o stimolazioni a un certo numero di sensi, non solo vista e udito. Riassumendo: se l'aggettivo immerso trova nel proprio sostantivo di riferimento il termine immersione, per contrasto l'aggettivo immersivo non potrà che rifarsi ad un termine differente, che indicheremo con il lemma immersività, già largamente attestato nella letteratura scientifica italiana.

Lo scarto nell'utilizzo del binomio immersivo-immersività rispetto al corrispettivo parente immerso-immersione non appare così soltanto un vezzo terminologico: viene confermata dalle desinenze -ivo e -ività dei primi due termini la forte componente attiva che li caratterizza. Possiamo dunque concludere che qualcosa di immersivo sia più coinvolgente

\footnotetext{
${ }^{1}$ Treccani, vocabolario online, voce immersivo:

http://www.treccani.it/vocabolario/immersivo_\%28Neologismi\%29/ (ultimo accesso 20/01/2022). II vocabolario ne attesta la presenza già nella stampa quotidiana nazionale del 1993 e lo inserisce nella lista dei neologismi dell'anno 2008.

2 Ibidem.

${ }^{3}$ Cambridge Dictionary, voce immersive: https://dictionary.cambridge.org/it/dizionario/inglese/immersive (ultimo accesso 20/01/2022) [traduzione mia].
} 
e stimolante di qualcosa di immerso, così come un'immersione sia un'azione che in qualche maniera risulterà sempre maggiormente passiva rispetto a una qualunque immersività. Non è un caso quindi che all'aggettivo "immersive" si associ direttamente il registro del contesto che l'ha visto nascere, quello dei media, del teatro e del cinema contemporanei, ambienti che - soprattutto nello specifico ambito sperimentale che ci interessa - vedono al loro interno il coinvolgimento di corpi e soggetti nella maniera più totalizzante possibile, non solo e non più in senso strettamente spettatoriale, ma anche e soprattutto attraverso un'esperienza più complicata, che pone volutamente il pubblico di fronte a delle scelte di fruizione, a delle possibilità attive e non più passive, sempre di natura immersiva.

Seguendo la traccia sopra esplicitata si è deciso di volgere lo sguardo verso una pratica di produzione artistica ben precisa: il Cinema VR. Tale scelta rispecchia infatti al meglio la natura ambigua dell'esperienza immersiva, permettendo di evidenziare diversi punti di forza e svariate criticità all'interno di una riflessione teorica complessa.

\section{Immersività mediata o non-mediata?}

La Virtual Reality non è di per sé ascrivibile allo sguardo e all'apparato cinematografico. La scelta di utilizzare il termine "Cinema VR" ci è utile per circoscrivere un insieme di caratteristiche. Con esso si vuole intendere quella recente tendenza cinematografica che vuole esplorare le potenzialità degli stilemi tipici della VR (in particolar modo la possibilità di visione tridimensionale a $360^{\circ}$ e di audio surround) trasportandoli nella narrativa filmica.

Tale tendenza del cinema contemporaneo, tecnicamente sperimentale, ha trovato di recente un vivo interesse ${ }^{4}$ all'interno di molti festival cinematografici internazionali di rilievo $^{5}$. Un esempio virtuoso in questo senso è costituito dall'inclusione nel 2017, all'interno della selezione ufficiale di Cannes 70, dell'installazione in realtà virtuale CARNE y ARENA (Virtually Present, Physically Invisible) ${ }^{6}$ del regista Alejandro González Iñárritu. Per quanto in questo caso, così come in altri prodotti nati dalla computer grafica, la

\footnotetext{
${ }^{4}$ A partire dalla sezione della Biennale Cinema di Venezia Venice VR Expanded, nata nel 2017 come Venice Virtual Reality, prima competizione di film in Realtà Virtuale in un festival cinematografico internazionale.

${ }^{5}$ I quali, pur spesso accostando prodotti legati al gaming e filmati tridimensionali in $360^{\circ}$ o dalla forte componente narrativa, hanno iniziato comunque a mostrare una forte apertura nei confronti di questo tipo di Cinema appena nato.

${ }^{6}$ A. G. Iñárritu, CARNE y ARENA (Virtually Present, Physically Invisible), Stati Uniti d'America 2017, in collaborazione con il direttore della fotografia Emmanuel Lubetzki, il compositore Alva Noto e gli ILMxLAB.
} 
differenza tra istallazione virtuale, performance multimediale e cinema sia molto più sottile che nel caso dei filmati tridimensionali in $360^{\circ}$, quello che ci preme qui evidenziare è l'attenzione che i due più prestigiosi festival smaccatamente cinematografici al mondo hanno iniziato a rivolgere nei confronti di produzioni simili.

Nel Cinema VR la componente mediale è apparentemente costituita dall'immagine cinematografica. Andrea Pinotti si è interrogato sulla questione, in seguito alla fruizione in prima persona del sopracitato CARNE y ARENA, arrivando a formulare la nozione di «an-icona», dalla quale lo studioso vede nascere anche una nuova "an-icologia"7 . Egli sostiene che le an-icone,

[i]n quanto tali, minano il paradigma dominante delle teorie occidentali sull'immagine (condivise dalla dottrina classica sulla mimesis, il rapporto fenomenologico coscienzaimmagine, le teorie analitiche della rappresentazione, gli approcci semiotici e iconologici) basate sul concetto di "re-presentazione". Questa nozione trascura il punto cruciale per quanto riguarda le an-icone: la sensazione di "presenza" associata all'abitare il mondo dell'immagine. "Presentificazione" anziché rappresentazione, è qui il problema chiave. I soggetti, rapportandosi con le an-icone, non sono più osservatori visivi di fronte a immagini isolate dal mondo reale mediante un dispositivo di inquadratura: essi diventano experiencers che vivono in un quasi-mondo, il quale offre stimoli multisensoriali e consente affordance e interazioni sensomotori. ${ }^{8}$

Secondo Pinotti lo spettatore diventa appunto "experiencer" in corrispondenza di precisi stimoli, vale a dire quando le immagini diventano ambienti forniti di "agencies" e "affordances" 9 , an-icone appunto. Questo avviene in quanto, in un'esperienza simile, «»

[g]li sforzi per implementare le stimolazioni multisensoriali vanno di pari passo con il tentativo di creare immagini che appaiano sempre meno distinguibili dalla realtà: immagini che negano sé stesse come "immagini di" qualcosa, che è a sua volta non un'immagine, ma la realtà stessa. ${ }^{10}$

\footnotetext{
${ }^{7}$ Le immagini "an-iconiche", seppur paradossali, «sfidano la condizione di insularità - tradizionalmente garantita da un dispositivo di inquadratura delimitante la tela o lo schermo - e di separatezza», proprietà intrinseca dell'immagine, che la rende tale, «al fine di istituire uno spazio-tempo che è compatibile e coerente con lo spazio-tempo reale». Esse creano «un complesso fenomeno esperienziale che mischia virtualità e incorporazione, partecipazione ed empatia» attraverso «[i]l campo visivo, completamente saturo come nella visione naturale [...]. Non ci sono bordi o cornici che possano aiutare a distinguere ciò che è dentro e ciò che è fuori dall'immagine. La sensazione di "presenza", di "essere lì" è molto intensa». Cfr. A. Pinotti, Self-Negating Images: Towards An-Iconology, intervento in occasione dell'International and Interdisciplinary Conference IMMAGINI? Image and Imagination between Representation, Communication, Education and Psychology, Bressanone (BZ), 27-28 novembre 2017, pubblicato il 13/12/2017, p. 3, [traduzione mia].

${ }^{8}$ Ibidem, [traduzione mia].

${ }^{9}$ Ivi, p. 3.

${ }^{10}$ Ivi, pp. 2-3, [traduzione mia, termini inglesi volutamente non tradotti].
} 
Utilizzando le tecnologie VR si otterrebbe secondo Pinotti un rovesciamento ontologico dell'immagine come medium, che in quanto an-icona porterebbe a percepire «un'apparente non-mediazione degli ambienti mediali immersivi» ${ }^{11}$, senza tuttavia stravolgere la narratività del mezzo.

\section{Immersività immediale e liveness}

II Cinema VR propone quindi al fruitore un'esperienza mediata o non-mediata? Piuttosto che cercare una risposta ben definita, è forse più utile evidenziare quanto espresso da Yves Citton in Immedialità intra-attiva e intermedialità estetica, dove egli osserva come sia opportuno concentrarsi

[...] su un'altra forma di intermedialità e di interazione, in cui siamo noi, i soggetti umani, a essere nello spazio inter-mediario. [...S]iamo dentro, immersi nelle circolazioni mediatiche - in medias res mediaticas - in uno stato allo stesso tempo perfettamente banale e perfettamente traumatico di im-medialità. ${ }^{12}$

La metafora, particolarmente efficace, è quella di un continuo «muoversi "attraverso" una folla in movimento ${ }^{13}$ :

siamo parte attiva di [...] intra-azioni: una disposizione, di cui facciamo parte, la quale agisce su se stessa attraverso di noi. Noi siamo uno degli intermediari di questa intraazione. Il rovesciamento dei ruoli è allora assai inquietante: noi diveniamo immagini, mentre le immagini divengono soggetti. Si parla spesso in questi casi di "illusione" ${ }^{14}$.

Il soggetto nello "spazio inter-mediario" - liminale, per dirla con Turner"15 - e la sua "immersione nella circolazioni mediatiche"; "intra-azione" e "illusione": sono tutti concetti applicabili coerentemente anche al Cinema VR. Questa particolare forma artistica im-mediale sembrerebbe così riconducibile anche alla definizione che Auslander dà della digital liveness:

\footnotetext{
${ }^{11}$ A. Pinotti, op. cit., pp. 2-3 [traduzione mia].

${ }^{12}$ Y. Citton, Immedialità intra-attiva e intermedialità estetica, in «Rivista di estetica», n. 63, pp. 99-120, https://journals.openedition.org/estetica/1289\#entries (ultimo accesso 20/01/2022)

${ }^{13}$ Ibidem. Un'idea che, per certi versi, richiamerebbe quella di prosumer-Cfr. A. Toffler, The Third Wave, Bantam Books, New York 1980 - in un network.

14 «Termine che bisogna intendere nel senso forte (e ubiquitario nel mondo sociale) che gli ha dato Pierre Bourdieu: I'illusio è il gioco (-ludus) in (in-) cui sono preso ben al di là del mio controllo, pur consapevole di stare giocando» (Ibidem).

${ }^{15}$ Cfr. V. Turner, Dal rito al teatro, il Mulino, Bologna 2014.
} 
liveness does not inhere in a technological artifact or its operations - it results from our engagement with it and our willingness to bring it into full presence for ourselves. We do not perceive interactive technologies as live because they respond to us in real time [...]. Rather, we perceive real-time response in some cases as a demand that concretizes a claim to liveness, a claim that we, the audience, must accept as binding upon us in order for it to be fulfilled. [...] Interactive technologies do not disclose themselves to us as live but become so only as a conscious achievement on our part. ${ }^{16}$

Se possiamo considerare il Cinema VR un'espressione artistica immersiva, quindi, possiamo anche considerarla im-mediale, nonché riconducibile alla digital liveness. Così facendo, avanzando lungo il filo della nostra proposta teorica, si delineano i nuclei centrali della riflessione attorno a ciò che fin qui abbiamo voluto definire Cinema VR: l'immagine o schermo o medium; il corpo o soggetto; e la vicendevole percezione di essi.

\section{Cinema VR come esperienza archi-schermica}

\section{Le «idee sensibili» di Merleau-Ponty e l' "archi-schermo» di Carbone}

Isolati i tre punti focali del discorso teorico, possiamo agilmente volgerci ad una proposta filosofica che li faccia propri, la quale nel ripensamento della fenomenologia di Merleau-Ponty attraverso l'estetica mediale contemporanea proposta in Filosofiaschermi. Dal cinema alla rivoluzione digitale ${ }^{17}$, permette a Mauro Carbone di sviluppare I'“idea sensibile” di archi-schermo ${ }^{18}$.

Per introdurre una riflessione così ampia, sembrerebbe opportuno apporre un'adeguata premessa. Walter Benjamin in L'opera d'arte nell'epoca della sua riproducibilità tecnica scrive:

Nel giro di lunghi periodi storici, insieme con le forme complessive di esistenza delle collettività umane, si modificano anche i modi e i generi della loro percezione sensoriale. Il modo e il genere secondo cui si organizza la percezione sensoriale umana - il medium in cui essa ha luogo - è condizionato non soltanto in senso naturale, bensì anche in senso storico. [...] avvicinare le cose spazialmente e umanamente è un'esigenza vivissima delle masse attuali, come lo è la loro tendenza al superamento dell'unicità di qualunque dato tramite la ricezione della sua riproduzione. Ogni giorno si fa valere in modo sempre più incontestabile il bisogno di impossessarsi dell'oggetto da una distanza il più possibile ravvicinata nell'immagine, o meglio nell'effigie, nella riproduzione. [...] L'adeguamento

\footnotetext{
${ }^{16}$ P. Auslander, Digital Liveness. A Historico-Philosophical Perspective, in «PAJ: A Journal of Performance and Art», vol. 34, n. 3, September 2012, p. 8.

${ }^{17}$ M. Carbone, Filosofia-schermi. Dal cinema alla rivoluzione digitale, Raffaello Cortina Editore, Milano 2016.

${ }^{18}$ Cfr. infra.
} 
della realtà alle masse e delle masse alla realtà è un processo di portata illimitata sia per il pensiero sia per la percezione ${ }^{19}$.

L'evoluzione della percezione, secondo l'approccio marxista sui generis del filosofo tedesco, avviene contestualmente alle modificazioni sociali di massa, attraverso il medium preponderante del periodo storico, il quale non è quindi solo un mezzo, ma anche e soprattutto un emblema percettivo della sua epoca di riferimento, che rispecchia a livello storico l'evoluzione della massa stessa. Secondo Benjamin l'esigenza dell'uomo contemporaneo, che si rispecchia all'interno del discorso del filosofo in modo particolare nella fotografia e nel Cinema della sua epoca, è quella di farsi più vicino al medium, tanto sensibilmente quanto socialmente, al fine di afferrare l'oggetto che esso veicola. Se pensiamo al periodo storico in cui queste idee vengono espresse - siamo tra il 1935 e il 1939 - non possiamo non attestarne la portata innovativa e precorritrice nei confronti dei due media da Benjamin considerati (all'epoca giovanissimi) e della loro valenza sociale, valida anche oggi nel nostro discorso se consideriamo, al loro posto, i digital media e, nello specifico caso che ci interessa, le esperienze immersive considerate. È sempre Benjamin, nel passo sopra riportato, a evidenziare quanto con l'evoluzione dei media, della loro valenza sociale e della loro percezione si verifichi anche un'evoluzione del pensiero: I'opera d'arte che apre a una nuova percezione, così, diventa terreno di riflessione e di messa in discussione del pensiero stesso, pensiero che attorno a simili cambiamenti (a esso pariteticamente esterni e interni) deve interrogarsi. Ed è proprio con questo spirito che Carbone propone un discorso sulla medialità e sul corpo come dispositivi di visione, così come sul loro rapporto con l'evoluzione del pensiero, analizzando nei fatti la stretta correlazione tra gli schermi (il vero grande medium a noi contemporaneo) e il pensiero filosofico, facendo proprie e portando avanti specialmente le ultime posizioni teoriche di Merleau-Ponty, originale esponente della fenomenologia francese. Le idee del filosofo francese, non del o sul Cinema, costituiscono infatti un pensiero che ripensa se stesso e il proprio stile di espressione in funzione del medium caratteristico della propria epoca, il cinema appunto. Merleau-Ponty sostiene che il cinema come la filosofia ponga al centro

\footnotetext{
${ }^{19}$ W. Benjamin, L'opera d'arte nell'epoca della sua riproducibilità tecnica [1936], Einaudi, Torino 2014, pp. 9-11, [corsivo mio].
} 
un'attenzione per il visibile, manifesta nel nostro rapporto col mondo e con gli altri, e che la percezione in tale processo, sia esso filosofico o cinematografico, ricopra il ruolo primario ${ }^{20}$. Per Carbone la fenomenologia giunge così a evidenziare quanto in questo processo giochi un ruolo fondamentale l'idea di precessione, essenza della visione stessa: il filosofo italiano evidenzia come il termine "precessione" voglia evidenziare un rapporto di «reciprocità» quale «movimento di mutua anticipazione tra i termini che sono implicati nella relazione», vale a dire le cose e lo sguardo, l'immaginario e il fattuale, dove «il primato dell'uno dei due termini sull'altro diventa indecidibile» ${ }^{21}$. Un'idea simile invita di conseguenza a ripensare la realtà alla radice. Essa «consente infatti di fare a meno di quella di un prima assoluto nello spazio e nel tempo (o addirittura di un "prima" dello spazio e del tempo)» ${ }^{22}$. Come ricorda sempre Carbone, uscendo da un tempo e uno spazio prestabiliti, secondo Merleau-Ponty, si entrava nel regno delle "idee sensibili", inseparabili cioè dalla loro presentazione sensibile ${ }^{23}$.

La portata rivoluzionaria di simili proposte teoriche non venne purtroppo raccolta in seguito alla prematura morte del fenomenologo francese. Le istanze critiche dell'ultimo Merleau-Ponty, però, che abbiamo visto schierarsi contro la posizione platonica che attraverso il mito della caverna ha influenzato tutta la filosofia successiva e che vedeva un contrapporsi tra «le ombre ingannevoli di ciò che appare e la luce pura della verità ${ }^{24}$, suggeriscono per Carbone quanto sia:

[...] evidente l'intento merleau-pontiano di operare una riabilitazione ontologica della superficie di mediazione con cui accompagnare il tendenziale affermarsi di un diverso modo di concepire il darsi dal vero, che da una configurazione teatrale - ossia, per antonomasia, rappresentativa, che s'inaugura con l'aprirsi del sipario - sembra passare a una cinematografica. Quella che detta la già evocata sequenza di una filosofia-cinema.

\footnotetext{
${ }^{20}$ Cfr. M. Carbone, op. cit., pp. 30-50.

${ }^{21}$ Ivi, p. 55.

22 Ivi, p. 56.

23 "“idee sensibili" [... alle quali] anziché una coesione dovuta al fatto che esse "racchiudono ciò che dicono", egli attribuisce loro "una coesione senza concetto, affine alla coesione delle parti del mio corpo o a quella del mio corpo e del mondo". [...] Più in generale, si tratta di idee che possiamo "esperire" - perché conoscerle significa farne fisicamente esperienza - solo incontrandole in una delle loro manifestazioni sensibili: incontrarle, potremmo dire, su o dietro qualche tipo di "schermo" o di "velo" (Merleau-Ponty usa qui indifferentemente i due termini), foss'anche quello metaforico costituito dall'ascolto di un brano musicale o dalla lettura di un'opera letteraria. La presenza di una superficie di mediazione si rivela comunque decisiva per questo pensiero senza concetto». Cfr. M. Carbone, op. cit., pp. 61-63.
}

24 Ivi, p. 67. 
Carbone si lancia così in una riconsiderazione storica della contemplazione di fronte a un tipo di zona quadrangolare delimitata, con la quale da sempre l'uomo predispone caratteri peculiari in rapporto con il suo sguardo ${ }^{25}$ arrivando allo schermo cinematografico il quale, seppure rispetto ad altri esempi viva di una contemplazione "diversamente declinata" 26 in fase di utilizzo, tuttavia in quanto oggetto-dispositivo ottico al pari deli altri, «conferi[sce] a quanto appare su quella superficie un valore che eccede il suo mero apparire. [In altre parole] quanto appare sullo schermo non è solo ciò che appare ${ }^{27}$. Un simile approccio, per Carbone, è rintracciabile anche in tempi più remoti, dove la delimitazione, seppure non strettamente rettangolare, era comunque spazialmente efficace ${ }^{28}$. Tale sforzo era volto a «celebrare l'enigma delle immagini stesse, nonché quello della superficie di volta in volta investita di tale celebrazione e perciò delimitata rispetto allo spazio circostante» ${ }^{29}$ : il filosofo italiano arriva così a definire tale superficie " schermo", intendendolo quale insieme delle condizioni di possibilità della mostrazione che nella nostra cultura sono state via via storicamente aperte dal corpo umano stesso ${ }^{30}$. Coerentemente alla riflessione condotta, per Carbone l'archi-schermo è un tema in rapporto di precessione con le proprie variazioni «pur non cessando di eccederle, a queste non essendo riducibile ma da esse risultando inseparabile e con il loro divenire potendo divenire a propria volta» ${ }^{31}$.

È proprio attraverso questo tema che Carbone tenta, se vogliamo, di perseguire l'obiettivo incompiuto di "sovvertire il platonismo", rileggendo il mito della caverna platonica e così rilanciando il discorso merleau-pontiano: egli evidenzia nei fatti come nel testo greco, oltre alla famosa "parete opposta" che funge da schermo di mostrazione, sia presente anche un parapetto, dispositivo di protezione dietro al quale si nascondono co-

\footnotetext{
${ }^{25}$ Dal templum (parola contenuta appunto nel verbo "contemplare" e che in origine indicava il rettangolo che gli aruspici romani disegnavano con il loro bastone nel cielo per vedervi il tipo di passaggio che le aquile avrebbero compiuto al suo interno), al tempio, alla tela pittorica.

${ }^{26} \mathrm{Cfr}$. riferimenti alle interpretazioni sul declino dell'aura benjaminiana: M. Carbone, op. cit., p. 98, nota 29.

27 Ibidem.

${ }^{28}$ Dal "recinto sacro" greco alla tenda pitagorica (che sovrasignifica il visibile vietandolo), indietro fino alle immagini rupestri della grotta di Chuvet dove «ebbe luogo un sofisticato sforzo collettivo per contemplare immagini in movimento, tale da indurre Werner Herzog a parlare "quasi di una forma di protocinema" e Marc Azéma di "una vera e propria 'preistoria' del cinema"». Ivi, p. 100.

${ }^{29}$ Ibidem.

30 Ibidem.

${ }^{31}$ Ivi, p. 101.
} 
loro i quali ergono sopra di esso gli oggetti che proiettano le loro ombre sulla parete precedente, ombre "selezionate" attraverso il nascondimento stesso. In questa sorta di Teatro delle ombre ante-litteram, è interessante notare come questo parapetto mostri e nasconda, assolvendo alla «doppia funzione caratteristica di un "archi-schermo" ${ }^{32}$ e permettendo all'autore di sostenere che già il mito platonico sottolinei la doppia significazione del termine e del tema sviluppato:

Sia come superficie di occultamento sia come superficie di mostrazione, l'archi-schermo sovradetermina lo spazio al quale è correlato. In un modo o nell'altro, esso presenta quindi più di se stesso, presenta eccedendo se stesso. In tal senso, è inevitabilmente uno schermo eccedente, che, per questa ragione, inevitabilmente sollecita il nostro desiderio in varie forme, promettendoci "sempre 'altro da vedere", , per dirla con Merleau-Ponty. [...] Ovviamente l'archi-schermo può segnare in diversi modi il carattere eccedente dello spazio cui è correlato: semplicemente delimitandolo, certo, o invece sovrapponendosi a esso, oppure combinando fra loro questi due elementi. Nel primo caso, lo spazio delimitato è sovradeterminato in senso positivo, laddove nel secondo lo è in senso negativo, giacché ci è proibito vederlo. ${ }^{33}$

Come Carbone stesso propone, una simile formulazione sembra sviluppare le ultime posizioni merleau-pontiane, le quali sembrano riferirsi a una vera e propria «esperienza primordiale dell'archi-schermo» ${ }^{34}$ :

Il carattere archi-schermico della nostra esperienza che emerge in questo passaggio risulta quindi fare tutt'uno col nostro corpo, vissuto come uno spazio (inter)posto nel sensibile di cui fa parte, tale da consentire d'intercettare perciò il sensibile stesso, conoscendo quest'ultimo e, per il suo tramite, quanto lo eccede. ${ }^{35}$

Questa intrinseca caratteristica di mostrazione eccedente dell'archi-schermo, è sempre riconducibile a un'esperienza corporea, e porta Carbone a concludere «non solo che non c'è visione senza archi-schermo, ma inoltre che quest'ultimo risulta, per il suo carattere eccedente, una superficie che istituisce relazioni». È proprio quest'ultima formulazione che definisce il valore spazio-temporale assoluto del tema: «benché ormai siano

\footnotetext{
${ }^{32}$ M. Carbone, op. cit., p. 104.

${ }^{33}$ Ivi, p. 105.

34 Ivi, p. 106.

${ }^{35}$ Ivi, p. 107.
} 
molti ad annunciare la sparizione prossima degli "schermi", io ritengo che questo non varrà invece per "I'archi-schermo" ॥ ${ }^{36}$, arriva a dichiarare il filosofo.

Non è difficile comprendere perché Carbone consideri gli schermi contemporanei la naturale manifestazione dell'archi-schermo e quindi il nostro dispositivo ottico di riferimento: dalla volontà di vedere al di là dello schermo alla volontà di vedere con (o secondo) lo schermo fino alla volontà concreta di entrarvi ${ }^{37}$, secondo un andamento progressivo per cui «l'ambiguità prima rintracciabile tra nascondere e mostrare tenderà a venir meno ${ }^{38}$, il pensiero esprimerebbe così se stesso attraverso una "filosofia archi-schermica"39.

A questo punto non dovrebbe apparire particolarmente complicato considerare il Cinema VR un'esperienza immersiva archi-schermica: se per il filosofo italiano l'archischermo è una nozione che si rifà alla medialità pur tuttavia superandola (o addirittura trascendendola), esso mostra e nasconde in maniera ambigua fin dalle origini della storia umana, e può essere ricondotto alla dimensione corporea del soggetto, che con esso e attraverso di esso si relaziona; esso è quindi una superficie non direttamente collegabile a un'estensione tecnica esterna, che pure ne è spesso una risultante. II Cinema VR nei fatti pone al centro un'esperienza percettiva estremamente corporea, la quale pur originandosi consistentemente dall'universo dei digital media, vive comunque in maniera paritetica di una conoscenza sensibile e primordiale del mondo: se è vero, infatti, che l'ambiguità tra il nascondere e il mostrare che vive l'archi-schermo tende ad assottigliarsi, nel Cinema VR sembrerebbe svilupparsi tale tendenza promettendo al corpo di diventare schermo, allo schermo di diventare corpo.

\section{Un corpo che diventa schermo, uno schermo che diventa corpo}

Sempre secondo Carbone già dal cinema delle origini, così come lungo tutta la loro storia in generale, gli schermi cinematografici generano seduzione nei confronti del pubblico. Essi contemplano da sempre l'esibizione del topos di uno spettatore talmente tan-

\footnotetext{
${ }^{36}$ M. Carbone, op. cit., p. 107.

${ }^{37}$ Ivi, pp. $115-116$.

${ }^{38}$ Ivi, p. 114.

${ }^{39}$ Particolarmente stimolante in questo senso è tutto il discorso che porta Carbone a sviluppare l'idea di «filosofia-schermi» come evoluzione della deleuziana "filosofia-cinema", che attraversa l'intero volume.
} 
to "avvinto dallo schermo da tentare di entrarvi» ${ }^{40}$, caratteristica intrinseca al medium seppure non sempre pienamente espressa:

invitare lo spettatore a vivere sullo schermo mi pare un elemento di seduzione inseparabile dall'esperienza filmica come tale, più in generale dall'esperienza del film quale viene vissuta, praticata e rielaborata dallo spettatore stesso: in fondo, anche gli innumerevoli miglioramenti tecnologici che hanno caratterizzato la storia del cinema possono essere considerati altrettanti tentativi per alimentare proprio quella seduzione. Ovviamente quest'ultima dovrà la sua efficacia anzitutto a quei caratteri dell'esperienza filmica che chiameremo "percettivo-affettivi", i quali finiranno per sedimentarsi nella corporeità stessa dello spettatore. ${ }^{41}$

II Cinema VR rilancia ulteriormente questa seduzione, insistendo proprio sulla sedimentazione visivo-corporea dei caratteri sopracitati. Una conferma in questo senso la si può scorgere poco più avanti, quando Carbone arriva ad applicare la nozione di "quasi soggetto" 42 alle immagini proposte e inseparabili dagli schermi a noi contemporanei, «"inanimat[i] ma interattiv[i]", laddove l'interattività sembra anzitutto caratterizzata quale forma particolare di interrelazione percettiva». Caratterizzante l'esperienza contemporanea con simili immagini è quindi «una particolare interattività percettiva, nonché affettiva»: attraverso di essa le immagini diverrebbero infatti «"quasi-agenti, delle pseudo-persone". [...] Come tali, essi producono un "quasi-agire" - un impersonale agency - con cui sfuggono alla tradizionale opposizione che assegna ai soggetti i caratteri dell'attività e agli oggetti quelli della passività». ${ }^{43}$

Questa capacità delle immagini e degli schermi, che potremmo definire "soggettuale", secondo Carbone non è però applicabile a una determinata tipologia di

\footnotetext{
${ }^{40}$ M. Carbone, op. cit., p. 120.

${ }^{41}$ Ivi, pp. 121-122.

${ }^{42}$ Carbone utilizza le riflessioni di Dufrenne a proposito dell' " "oggetto estetico", che - egli sottolinea - si dà come oggetto in primo luogo sensibile cui risulta essenziale, per trovare il proprio compimento, la percezione dello spettatore. La correlazione tra questi e il proprio oggetto - spiega Dufrenne - ha il suo stadio originario nella "presenza", in cui la corporeità dell'uno e la sensibilità dell'altro compongono una totalità "indiscernibile". [...] L'oggetto estetico si rivelerà allora dotato di un proprio "mondo", con la sua particolare struttura sensibile e affettiva. Per questo Dufrenne definisce l'oggetto estetico come un "quasi soggetto" ». Ibidem.

${ }^{43}$ Seppure più che di «presenza» Carbone preferisca parlare, sulla scia dei lemmi sopracitati e di Dufrenne stesso, di "“quasi-presenza", poiché "l'immaginazione" insinua già in tale esperienza uno sfondo di [...] "visibilità imminente", che quindi non potrà se non eccedere ogni presenza», tuttavia le immagini schermiche prese in considerazione «[c]on questo particolare statuto, si iscrivono immediatamente nella nostra correlazione sensibile-affettiva con il mondo, già percorsa da un pensare che a sua volta opera al di qua della distinzione tra attività e passività» (Ivi, pp. 124-125. Nello specifico cfr. i riferimenti a Turkle e Mitchell, note 17 e 18).
} 
schermo piuttosto che a un'altra, in questo senso essa sarebbe quindi costitutiva degli archi-schermi, caratterizzati ontologicamente da una promessa di reversibilità:

è proprio in nome di tale reversibilità che gli schermi possono promettere (ma anche minacciare) di farci vivere al loro interno. Si delinea così una sorta di chiasma in virtù del quale, se la correlazione con lo spettatore costituisce l'archi-schermo come "quasisoggetto", di rimando quest'ultimo costituisce lo spettatore come "quasi-immagine". A ben guardare, sono questi i termini della questione dell'immersività. ${ }^{44}$

L'immagine è divenuta soggetto e viceversa, il corpo è divenuto schermo, l'immersività cinematografica è apparentemente avvenuta. Certo, ciò resta comunque una realizzazione mai del tutto effettiva: il fruitore resta uno spettatore vedente e visibile, l'immagine non abita al posto del soggetto restando tecnicamente sullo schermo, in altre parole ha ragione Carbone quando sostiene che "stiamo parlando di "una reversibilità sempre imminente e mai realizzata di fatto" o di una "visibilità immanente"»; che sarebbe

proprio grazie a questa costitutiva immanenza che gli schermi possono suggerire la perturbante promessa di farci vivere al loro interno [...] senza tuttavia arrivare a mantenerla, ossia senza giungere a con-fondere i due poli - vedente e visibile - di quella reversibilità che, realizzandosi pienamente, finirebbe per impedire la visione stessa[.] $]^{45}$

Va però evidenziato come la possibilità che una simile esperienza avvenga prima o poi (o che effettivamente avvenga già a livello percettivo) rimane: l'esperienza immersiva del Cinema VR sembrerebbe se non riuscire, quantomeno paradossalmente potenziare enormemente l'intento di farci vivere all'interno di uno schermo, mantenendo la promessa sopracitata, pur tuttavia senza fondere i poli del vedente e del visibile, senza comunque realizzare quindi una piena reversibilità a scapito della visione e della percezione, che in ogni caso vedono in questa nuova forma espressiva una piega in gran parte originale. Nella relazione sensibile tra il soggetto e l'opera immersiva stessa, instaurata e creata attraverso il proprio corpo, non pare così azzardato rintracciare i caratteri fondamentali della superficie istitutrice di relazioni che sarebbe l'archi-schermo con il suo ca-

\footnotetext{
${ }^{44}$ M. Carbone, op. cit., p. 128.

45 Ivi, p. 129.
} 
rattere eccedente: un carattere che come abbiamo visto, nel caso del Cinema VR particolarmente, fa "tutt'uno col nostro corpo». ${ }^{46}$

\section{Il soggetto nel Cinema VR}

\section{VR e Cinema}

Abbiamo cercato di proporre più sopra un'idea di Cinema VR, intendendolo come nuova forma artistica all'interno della quale si ritrovano tecniche e stilemi della VR affiancati da intenti narrativi prettamente cinematografici: nello sviluppo della VR la creazione di ambienti tridimensionali costituisce da sempre il punto di partenza, essendo la caratteristica principale ${ }^{47}$ dei sistemi di grafica immersivi insieme al ruolo centrale svolto dal corpo nella fruizione dell'esperienza, come testimonia Lanier ${ }^{48}$. Egli definisce il «dispositivo ideale di realtà virtuale come [...] uno specchio senso-motorio, oppure [...] un rovesciamento del corpo umano ${ }^{49}$. La conoscenza che deriva dall'esperienza della VR però non è mai solo fisica. Essa è infatti «una tecnologia mediale che pone al primo posto la stimolazione non di un ambiente alternativo, ma delle dinamiche cognitive in base alle quali si percepisce il mondo ${ }^{50}$. II discorso di Lanier, tanto scientifico quanto estetico, avvicina così alcune evidenziazioni del medium considerato all'ambito cinematografico già delineato, dove la VR vive di una forte intenzione narrativa, manifesta in maniera univoca nei filmati tridimensionali a $360^{\circ} .{ }^{51}$ In simili casi, caratterizzati dall'evidente referenzialità delle immagini, gli spettatori sono votati ad una fruizione apparentemente più passiva, che non permette di decidere lo spostamento del proprio corpo, ma solo della propria visuale rispetto

\footnotetext{
${ }^{46}$ L'opera cinematografica non si osserva soltanto, attraverso uno schermo stroboscopico come quello del visore, ma si vive sul proprio corpo, vale a dire dove l'archi-schermo stesso che permette la visione è il corpo.

${ }^{47}$ Oltre all'utilizzo di un visore (non ci interessa qui affrontare discorsi più ampi sui cosiddetti Virtual Enviroments multiutente e su esempi di CAVEs o simili, che richiederebbero una trattazione adeguata e differente).

${ }^{48}$ Cfr. J. Lanier, L'alba del nuovo tutto. Il futuro della realtà virtuale, II Saggiatore, Milano 2019 per un'esaustiva e originale trattazione della storia della VR e dei suoi inventori, in particolare qui p. 61.

${ }^{49}$ Ibidem.

50 Ivi, p. 210. Cfr. anche p. 70: «Grazie alla realtà virtuale impariamo a percepire ciò che rende reale la realtà fisica. Impariamo a eseguire nuove indagini sperimentali con i nostri corpi e le nostre menti, momento per momento, in maniera perlopiù inconscia. L'incontro con la realtà virtuale al massimo livello raffina la nostra capacità di discernere la realtà fisica e di immergerci in essa».

${ }^{51}$ Seppure si possano inserire all'interno della categoria Cinema VR sia produzioni di questo tipo sia video interamente digitali frutto di un lavoro di computer grafica molto accurato, in entrambe le tipologie i prodotti, per appartenere alla categoria, devono essere caratterizzati dalla tridimensionalità a $360^{\circ}$, fruibili per mezzo di visori VR, nonché essere contraddistinti da una forte narratività, preponderante rispetto alla componente interattiva.
} 
all'immagine dinamica del filmato, muovendo il capo. Nonostante ciò, e anzi forse proprio per questo, tali prodotti si prestano ad una narratività estremamente maggiore: troviamo infatti in questa sottocategoria prodotti che ci sembrano più spiccatamente cinematografici, pur mantenendo comunque quella libertà di scelta nella fruizione che abbiamo visto associata all'idea di immersività, nonché quella conoscenza corporea e sensibile col mondo di natura archi-schermica precedentemente proposta.

\section{Virtualità e fisicità, presenza e invisibilità}

Alla luce di quanto detto finora, proviamo ad analizzare nella sua complessità il Cinema VR. Per fare ciò torniamo al caso di studio che ci ha accompagnato finora, vale a dire CARNE y ARENA (Virtually Present, Physically Invisible) di Alejandro G. Iñárritu, avvalendoci in particolar modo delle riflessioni di Adriano D'Aloia a riguardo.

Come appare evidente fin dal suo sottotitolo, l'opera del regista messicano vive di una natura apparentemente duplice, che rende il fruitore, contemporaneamente, «virtualmente presente, fisicamente invisibile». D'Aloia sostiene che l'espressione

[...] dovrebbe essere rivolta riflessivamente: si è fisicamente invisibili non solo ai personaggi, con cui non si interagisce concretamente in alcun modo, ma anche a sé stessi. [...] Ci si trova invece in un ambiente e in un'esperienza in cui la partecipazione e la sensibilità corporea sono sollecitate (certamente più di quanto non lo siano al cinema), ma al contempo denegate da una paradossale invisibilità. ${ }^{52}$

Se l'autore vuole evidenziare con simili osservazioni come tale natura fruitiva si risolva in un paradosso, d'altra parte Pinotti sottolinea giustamente come ad essa corrisponda «la logica dell'“immediatezza trasparente” descritta da Bolter e Grusin: «un'apparente non mediazione degli ambienti mediali immersivi che si ottiene attraverso l'impiego di mediazioni tecnologiche altamente sofisticate ${ }^{53}$, posizione questa che trova nel testo dei due autori statunitensi una conferma diretta. Come segnala Citton ${ }^{54}$ sono infatti gli stessi Bolter e Grusin, all'interno dell'imprescindibile Remediation: Understanding New Media del 1999 a

\footnotetext{
${ }^{52}$ A. D'Aloia, Virtualmente presente, fisicamente invisibile, in «Fata Morgana WEB», 08/01/2018, www.fatamorganaweb.it (ultimo accesso 20/01/2022).

${ }^{53}$ A. Pinotti, op. cit., p. 3, [traduzione mia].

${ }^{54}$ Y. Citton, op. cit. (ultimo accesso 20/01/2022).
} 
chiamare in causa proprio la VR: «la realtà virtuale è l'esempio più chiaro (e più trasparente!) di logica dell'immediatezza trasparente ${ }^{55}$. Ovviamente va riconosciuto a D'Aloia come Bolter e Grusin sembrino riferirsi a un modello ideale di VR, mentre CARNE y ARENA, così come altri prodotti similari, soffre inevitabilmente di alcuni limiti fruitivo-percettivi che ne tradiscono la forte medialità, evidenziati da D'Aloia stesso ${ }^{56}$. Secondo lo studioso il movimento da una parte e l'immaterialità unita alla bassa verosimiglianza degli attori virtuali dall'altra rischiano di minare la piena godibilità dell'esperienza immersiva. Va però evidenziato come la passività spettatoriale, notevolmente ridotta ma comunque presente (come sottolinea lo stesso D'Aloia rifacendosi a Montani), costituisca soprattutto in forme più prettamente cinematografiche di Cinema VR - quelle cioè che abbiamo associato a filmati referenziali tridimensionali a $360^{\circ}$ - de facto la parte più cinematografica dell'esperienza, senza per questo inficiarne l'immersività: in prodotti come Battle Hymn ${ }^{57}$, Daughters of Chibok ${ }^{58}$, Only the mountain remains $[5 \times 1 \text { Project }]^{59}$, Senegal Detour ${ }^{60}$, The Making Of [5×1 Project ${ }^{61}$, The Waiting Room ${ }^{62}$, Traveling While Black ${ }^{63}$, VR Free ${ }^{64}$, tutti selezionati nell'edizione 2019 di Venice Virtual Reality eccetto il secondo, si riscontra, oltre alla già citata verosimiglianza visiva assoluta (nei casi di prodotti a tecnica mista, ovviamente, riferibile alla sola parte filmata), un'assenza della possibilità di scelta nei movimenti del fruitore.

Quello che a primo acchito potrebbe sembrare un grosso limite, è invece a nostro avviso un punto di forza: se infatti in questo modo la componente interattiva coinvolge solamen-

\footnotetext{
55 J. D. Bolter, R. Grusin, Remediation. Competizione e integrazione tra media vecchi e nuovi, Guerini, Milano 2002, p. 191.

56 «Per quanto la libertà di movimento sulla scena sia funzionale a stabilire una relazione empatica con i migranti, alcuni aspetti riducono sensibilmente l'efficacia di questa tipologia di aggancio emotivo, anch'essi collegati alla paradossale componente corporea dell'esperienza. In primo luogo, le concrete sembianze dei personaggi: sono poco realistici e più simili agli avatar dei videogame o dei mondi virtuali, pur se percepiti nella loro tridimensionalità plastica. In secondo luogo, il fatto che, proprio in virtù della mobilità concessa al visitatore, è possibile avvicinarsi a tal punto agli avatar da poterli toccare... se non che li si scopre inconsistenti e li si può letteralmente attraversare - scoperta straniante che innesca non solo nuovamente un conflitto tra percezione tattile e percezione visiva, ma anche una contraddizione tra effettività emotiva e assenza materiale dell'Altro». Cfr. A. D'Aloia, op. cit.

${ }^{57}$ Y. Agmon, Battle Hymn, Israele 2019.

58 J.K. Benson, Daughters of Chibok, Nigeria 2019.

${ }^{59}$ W.-L. Chiang, Only the mountain remains [5×1 Project], Taiwan 2018.

${ }^{60}$ S. Sburlati, Senegal Detour, Italia 2019.

${ }^{61}$ Midi Z, The Making Of [5×1 Project], Taiwan 2019.

62 V. Mapplebeck, The Waiting Room, Regno Unito 2019.

${ }^{63}$ R.R. Williams, A. Nadarajah, F. Lajeunesse, P. Raphaël, Traveling While Black, Stati Uniti d'America e Canada 2019.

${ }^{64}$ M. Tangshir, VR Free, Italia 2019.
} 
te la sfera emotiva (come del resto avviene nel Cinema tradizionale), d'altra parte il coinvolgimento scaturito dalla referenzialità delle immagini è totale. Molto spesso il movimento è presente, un movimento di macchina che invece di inficiare la visione omnidirezionale la amplifica: ci lasciamo così trasportare fisicamente all'interno della scena, concentrando la nostra attenzione (e quindi la nostra possibilità di scelta) su personaggi, panorami, oggetti, dettagli che più ci attirano. Fisicamente, si badi, non significa per forza di cose corporalmente, il cortocircuito tra il proprio corpo seduto e la propria (non-)corporeità virtuale rimane, ma invece che smaterializzarci è come se esso ci moltiplicasse, amplificando il nostro coinvolgimento e non facendoci sentire "persi" e "imprigionati" nel mondo reale-virtuale. Alcuni degli esempi citati, come ad esempio Battle Hymn, esasperano ulteriormente questo gioco di corpi, consegnandoci addirittura (ed è ancora fondamentale evidenziare la referenzialità massima di queste immagini) un nuovo corpo che si muove e compie azioni al nostro posto, mediante una visuale in prima persona certamente disturbante, ma che rilancia l'immersività come esperienza archi-schermica all'ennesima potenza.

Germano Celant afferma a proposito di CARNE y ARENA (ma un discorso simile vale anche per i lavori da noi appena indicati):

Iñárritu rende osmotico lo scambio tra visione ed esperienza, nel quale si dissolve la dualità tra corpo organico e corpo artificiale. Nasce una fusione d'identità: un'unità psicofisica dove, varcando la soglia del virtuale, l'umano sconfina nell'immaginario e viceversa. È una rivoluzione comunicativa in cui il vedere si trasforma in sentire e in condividere fisicamente il cinema: una transizione dallo schermo allo sguardo dell'essere umano, con un'immersione totale dei sensi. ${ }^{65}$

Se risulta doveroso notare come nell'esperienza del Cinema VR permanga comunque una componente preponderante d'osservazione ${ }^{66}$, d'altra parte appare evidente anche l'interesse che il regista messicano nutre per l'ampliamento dello spazio, attraverso la caratteristica estetica della continuità, in solo apparente contrasto con il montaggio

\footnotetext{
${ }^{65}$ Comunicato stampa della Fondazione Prada di Milano per la presentazione ufficiale di CARNE y ARENA (Virtually Present, Physically Invisible): http://www.fondazioneprada.org/wp-content/uploads/1-Carne-yArena_Cannes_comunicato-stampa.pdf (ultimo accesso: 20/01/2022).

${ }^{66}$ Nonostante lo stesso Iñárritu durante la presentazione ufficiale dell'opera, abbia motivato le proprie scelte di natura tecnica con le seguenti parole: "La mia intenzione era di sperimentare con la tecnologia VR per esplorare la condizione umana e superare la dittatura dell'inquadratura, attraverso la quale le cose possono essere solo osservate, e reclamare lo spazio necessario al visitatore per vivere un'esperienza diretta nei panni degli immigrati, sotto la loro pelle e dentro i loro cuori». Ibidem.
} 
cinematografico. D’Aloia evidenzia infatti come attraverso un' «estensione dei mutevoli confini del "filmico" [...] l'apertura dell'orizzonte di visione a $360^{\circ}$ mett[a] in crisi alcuni dei parametri di base della visione filmica: i bordi dell'inquadratura, l'univocità del punto di vista, la guida eterodiretta dell'attenzione tramite il montaggio, le angolazioni e i movimenti di macchina ${ }^{67}$. Tuttavia lo studioso sostiene anche che in alcuni momenti della narrazione il «montaggio non si vede (fisicamente invisibile), ma c'è (virtualmente presente), poiché la rappresentazione sfrutta il richiamo attenzionale dello spettatore su un certo fronte per inserire oggetti o nuove situazioni sull'altro»: in questo modo sono ancora ravvisabili «dei "tagli", per quanto invisibili, che rompono la continuità del "piano sequenza", e continua a esistere un fuoricampo, il cui dinamismo anzi aumenta la centralità di ciò che inevitabilmente è escluso dal campo visivo. ${ }^{68}$

Quanto descritto da D’Aloia vale anche e soprattutto per i prodotti filmati dalla referenzialità elevata o assoluta: una condizione tecnica del genere apre a scenari esteticonarrativi sicuramente inediti per il medium cinematografico.

D’Aloia, a conclusione della sua analisi, riassume e rimarca la natura dell'esperienza immersiva con toni apparentemente negativi:

quest'esperienza, se analizzata fenomenologicamente, è soggetta a una dinamica più volte paradossale. In primo luogo è un'esperienza ipercorporea ma incorporea: per quanto investa letteralmente la corporeità dello spettatore, la eclissa nel suo stesso dispositivo, generando un conflitto tra stimoli tattili e visivi. In secondo luogo è un'esperienza intersoggettiva ma autosoggettiva: mentre lo spettatore c'è ma non si vede, il personaggio si vede ma non c'è, ed è proprio la possibilità della sua confutabilità fisica a interferire con l'empatia. In terzo luogo, è un'esperienza aschermica ma archi-schermica (per usare un'espressione di Mauro Carbone): I'estensione potenzialmente totale del campo visivo e l'apparente sospensione della sintassi formale della rappresentazione fanno corrispondere l'articolazione linguistica dell'opera agli stessi processi percettivi dello spettatore, ma le funzioni originarie dello schermo continuano a valere. ${ }^{69}$

\footnotetext{
${ }^{67}$ "Lo schermo si è avvicinato talmente agli occhi da formare con la testa dello spettatore una sorta di "camera oscura" ed essere assorbito in sé stesso. Lo spettatore si muove all'interno di uno spazio-schermo totale che appare una sua proiezione psichica. Questa sorta di "spettatore intradiegetico" si trova a dover organizzare in proprio un testo apertissimo, monta e muove da sé la sequenza, con l'impressione di incarnare l'istanza enunciativa del testo che invece nel cinema stabilisce preventivamente una prospettiva univoca e immutabile». Cfr. A. D'Aloia, op. cit.

68 Ibidem.

69 Ibidem.
} 
Per quanto a livello terminologico il discorso sia ineccepibile, il punto debole del passaggio sopra esposto risiede nel contrapporre avversativamente i termini opposti, non riuscendo nell'intento di cogliere appieno il fatto che il Cinema VR, nascendo appunto dalla fusione dei due ambiti da cui trae il nome, riesca anche a fondere - certo, paradossalmente, su questo D'Aloia ha ragione - caratteristiche apparentemente contraddittorie, generando un nuovo medium artistico: esso è infatti un'esperienza ipercorporea e incorporea, intersoggettiva e autosoggettiva, aschermica e, proprio per questo, archischermica. Solo in questo modo il Cinema VR può essere considerato a pieno titolo una nuova forma artistica (immersiva, im-mediale, archi-schermica): a riprova di ciò potrebbero acquistare un nuovo significato alcune ulteriori parole di Benjamin:

Così, la rappresentazione filmica della realtà è perciò incomparabilmente più significativa per l'uomo odierno, poiché, precisamente a motivo della sua penetrazione massimamente intensa mediante l'apparecchiatura, gli offre quell'aspetto, libero dall'apparecchiatura, che egli è legittimato a pretendere dall'opera d'arte. ${ }^{70}$

Sembrerebbe su questa stessa linea Carbone, quando argomenta:

la coscienza, concepita (appunto fenomenologicamente) quale coscienza corporea, trova la sua specifica forma storica anzitutto nel rapporto con i media percettivi ed espressivi che in quell'epoca ne assicurano le relazioni col mondo e con gli altri [...] indica[ndo] dunque l'intima compenetrazione tra un dispositivo ottico di riferimento, una determinata immagine della visione, una certa immagine del pensiero e un peculiare "processo di individuazione che si esercita su gruppi o su persone" ${ }^{\text {"11. }}$

Tuttavia il filosofo italiano in seguito osserva che "poiché l'“individuazione" incessantemente diviene l'altro lato di una delle molteplici relazioni che ci intessono, essa non può che perdere ogni implicito rinvio a un residuo indivisibile e deve perciò essere concepita piuttosto come dividuazione ${ }^{72}$. Sicuramente il Cinema VR rispetto al Cinema tradizionale svolge gran parte del suo meccanismo esperienziale attraverso la «dividualità», più ancora che l'individualità: seppure ciò non entri in contraddizione con quanto espresso più sopra, è inevitabile ravvisare uno scarto paradossale, anch'esso verosimilmente riconducibile all'influenza dei digital media contemporanei, che potrebbe compromette-

\footnotetext{
${ }^{70}$ W. Benjamin, op. cit., p. 26.

${ }^{71}$ M. Carbone, op. cit., p. 111.

72 Ivi, pp. 154-155.
} 
re in maniera determinante l'evoluzione artistica e sociale del Cinema VR. Benjamin del resto aveva ravvisato proprio nella collettivizzazione del Cinema il punto centrale della desacralizzazione dell'arte, che aveva permesso di introdurre la componente corporea nella fruizione e la conseguente sua politicizzazione: «ll cinema ha aperto una breccia nell'antica verità eraclitea - i desti hanno il loro mondo in comune, i dormienti ne hanno ciascuno uno per sé». È un tema cogente per chi si avvicina allo studio dell”“idea sensibile" di immersività, che riguarda particolarmente il Cinema VR e che merita di essere affrontato opportunamente in futuro.

\section{Conclusioni}

All'interno del presente saggio, dopo una breve premessa etimologica attorno all'idea di immersività, utile a circoscrivere il campo d'indagine, abbiamo introdotto il Cinema VR in qualità di oggetto di studio privilegiato, cercando di evidenziarne fin da subito la natura mediale ambigua, che permette di ricondurre questa particolare dimensione artistica ad una espressione im-mediale della digital liveness. Successivamente, soprattutto (ma non solo) attraverso la proposta avanzata da Mauro Carbone e dalla sua "filosofia-schermi", siamo riusciti ad associare al Cinema VR I'“idea sensibile" di "archi-schermo", manifesta nell'apparente vicendevole esperienza percettiva tra il corpo (o soggetto) e lo schermo (o immagine, o medium). Infine, dopo alcune precisazioni di carattere tecnico a proposito della Virtual Reality, si è tentato di rilanciare il discorso teorico avvalendosi anche dell'analisi di CARNE y ARENA (Virtually Present, Physically Invisible) condotta da Adriano D’Aloia, al fine di esplicitare quanto da noi sostenuto applicandolo sia nei confronti di un caso di studio specifico sia verso gli spunti critici che intorno ad esso sono nati, evidenziandone da ultimo anche possibili ampliamenti futuri attorno al tema della "dividualità".

\section{Bibliografia}

P. Auslander, Digital Liveness. A Historico-Philosophical Perspective, in «PAJ: A Journal of Performance and Art», vol. 34, n. 3, 2012, pp. 3-11.

W. Benjamin, L'opera d'arte nell'epoca della sua riproducibilità tecnica [1936], Einaudi, Torino 2014. 
J. D. Bolter, R. Grusin, Remediation. Competizione e integrazione tra media vecchi e nuovi, Guerini, Milano 2002.

M. Carbone, Filosofia-schermi. Dal cinema alla rivoluzione digitale, Raffaello Cortina Editore, Milano 2016.

Y. Citton, Immedialità intra-attiva e intermedialità estetica, in «Rivista di estetica», n. 63, 2016, pp. 99-120.

A. D'Aloia, Virtualmente presente, fisicamente invisibile, 08/01/2018, in "Fata Morgana WEB», 8 gennaio 2018: https://www.fatamorganaweb.it/virtualmente-presentefisicamente-invisibile-carne-y-arena-alejandro-inarritu (ultimo accesso 20/01/2022).

J. Lanier, L'alba del nuovo tutto. Il futuro della realtà virtuale, II Saggiatore, Milano 2019.

J. Machon, Immersive Theatres: Intimacy and Immediacy in Contemporary Performance, Palgrave Macmillan, London 2013.

A. Pinotti, Self-Negating Images: Towards An-Iconology, in «Multidisciplinary Digital Publishing Institute Proceedings», vol. 1, n. 9, 2017, p. 856.

V. Turner, Dal rito al teatro, il Mulino, Bologna 2014.

\section{Sitografia}

Treccani, vocabolario online, voce Immersivo:

http://www.treccani.it/vocabolario/immersivo_\%28Neologismi\%29/ (ultimo accesso 20/01/2022).

Cambridge Dictionary, voce Immersive:

https://dictionary.cambridge.org/it/dizionario/inglese/immersive (ultimo accesso 20/01/2022).

Comunicato stampa della Fondazione Prada di Milano per la presentazione ufficiale di CARNE y ARENA (Virtually Present, Physically Invisible): http://www.fondazioneprada.org/wp-content/uploads/1-Carne-yArena_Cannes_comunicato-stampa.pdf (ultimo accesso 20/01/2022).

\section{Biografia dell'autore/ Author's biography}

Francesco Melchiorri è ricercatore indipendente. Nel 2020 ha conseguito la Laurea Magistrale in Filologia Moderna. Scienze della Letteratura, del Teatro e del Cinema presso I'Università degli Studi di Pavia, dopo un periodo come visiting student presso il Corpus Christi College dell'Università di Cambridge. È ex-alunno dell'Almo Collegio Borromeo di Pavia, dove ha anche insegnato Lingua e Cultura Italiana L2 fino al 2021. Il suo principale campo di ricerca è il ruolo dei digital media nell'evoluzione del Cinema, del Teatro e della Performance, in particolare il rapporto tra digitalità e Teatro e tra Realtà Virtuale e Cinema. È stato Evaluation and Research Manager per il progetto "DanceMe UP», pro- 
getto del programma Europa Creativa incentrato sui media digitali e la danza contemporanea, e assistente alla regia volontario nello spettacolo Sei personaggi in cerca d'autore di Luigi Pirandello (2019) di Michele Sinisi. Attualmente è caporedattore di Birdmen Magazine. Insegna Italiano, Storia e Geografia nelle scuole secondarie di primo e secondo grado e collabora a diversi progetti teatrali.

Francesco Melchiorri is an independent researcher, based in Italy. In 2020 he obtained his Master's Degree in Modern Philology. Science of Literature, Theatre, Cinema from the University of Pavia, after a period as visiting student at the Corpus Christi College of the University of Cambridge. He is an alumnus of Almo Collegio Borromeo in Pavia, there he taught Italian Language and Culture for foreign students until 2021. His main field of research is the role of digital media in the evolution of Cinema, Theatre and Art Performances, in particular the relation between digitality and Theatre, and between Virtual Reality and Cinema. He has experience as Evaluation and Research Manager for the project "DanceMe UP», a Creative Europe project focused on digital media and contemporary dance, and as volunteer assistant director in the play Sei personaggi in cerca d'autore di Luigi Pirandello (2019) by Michele Sinisi. Currently, he is editor-in-chief for "Birdmen Magazine». He teaches Italian, History and Geography in lower and upper secondary schools, and collaborates in different theatrical projects.

Articolo sottoposto a double-blind peer review 